\title{
Installation of Lau Interferometer into X-Ray Microscope system for Phase-CT Measurement
}

$\underline{\text { Hidekazu Takano }}^{1}$, Yukinori Nagatani ${ }^{2}$, Songzhe Lian ${ }^{3}$, Koh Hashimoto ${ }^{1}$, Yanlin Wu ${ }^{1}$, and Atsushi Momose $^{1}$

1. Institute of Multidisciplinary Research for Advanced Materials, Tohoku University, Sendai, Japan

2. National Institute for Physiological Sciences, Okazaki, Japan

3. Faculty of Engineering, Information and Systems, University of Tsukuba, Tsukuba, Japan 'hidekazu.takano.c3@tohoku.ac.jp

Phase shift of X-ray wave in passing through a matter has been widely used for high-sensitive X-ray imaging. Although, there are various methodologies for the phase shift quantitation, grating interferometry is one of the most popular methods coupling with X-ray imaging. Quantitative X-ray phase imaging provides accurate three-dimensional measurement using computed tomography (CT). In order to realize a high spatial resolution, grating interferometry can be combined with an X-ray microscope system [1]. This concept can be applied to laboratory microscope with an incoherent X-ray source [2]. Based on this, we are developing a practical high-performance system for X-ray phase CT in laboratory.

A ZEISS Xradia 800 Ultra that operates with a spatial resolution of $50 \mathrm{~nm}$ was adopted as a base X-ray microscope system for installing a grating interferometer. The grating interferometer consisting of an absorption grating and a $\pi / 2$ phase grating was installed so that the gratings compose a Lau interferometer; the absorption grating functions as a source array to generate self-image of the phase grating. Parameters and the layout of the grating are described elsewhere [3].

In this combined system, a magnified self-image of the phase grating can be recorded with $\sim 60 \%$ visibility. The image produced by a phase stepping measurement is "twin phase image", where phase images with opposite contrast overlap with a certain distance. Figure 1 show images of a resolution test chart with finest line-and-space width of $50 \mathrm{~nm}$, obtained as an absorption image and a twin phase image. The spatial resolution of twin phase image is the same with the absorption image. Figure 2 shows comparison between an absorption image and a twin phase image of polystyrene spheres $3 \mu \mathrm{m}$ in diameter. The twin phase image can be converted to a quantitative phase image by deconvolution calculation. Although, there is a problem about artifact generation in a resulting image, phase-CT measurement was tested, as shown in Fig. 3 with polystyrene spheres on a plastic fiber.

We are improving the deconvolution procedure by introducing iterative process for optimizing the deconvolution function to moderate the artifact problem. We will show intrinsic performance of the system in near future and apply this system to various applications revealing inner structure in high spatial resolution.

\section{References:}

[1] W. Yashiro et al., Phys Rev Lett. 103, (2009) 180801.

[2] H. Kuwabara et al., Appl. Phys. Express 4, (2011) 062502.

[3] H. Takano et al., Proc. SPIE 10391, (2017) 1039110. 
[4] This work was supported by JST ERATO (grant No. JPMJER1403). The authors thank Carl Zeiss XRay Microscopy Inc. (Pleasanton, CA, USA) for help in installing the grating interferometer into the ZEISS Xradia 800 Ultra X-ray microscope

(a)

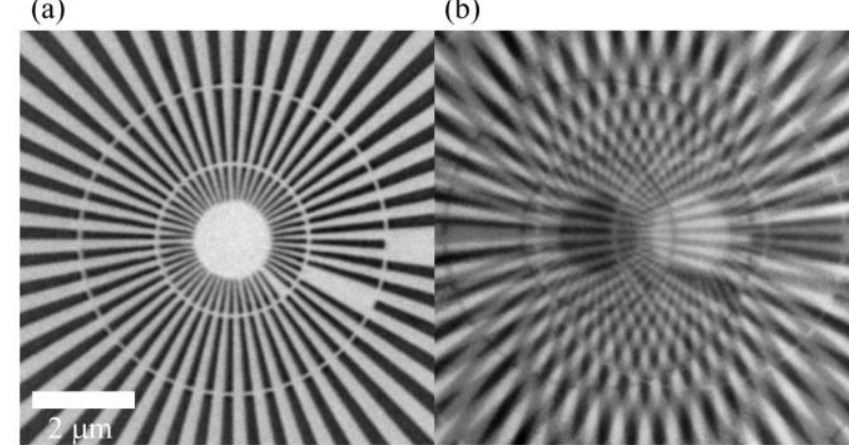

Figure. 1. Observed image of a resolution test chart with the innermost line width of $50 \mathrm{~nm}$. (a) Absorption image and (b) twin phase image. The gray scales of the image correspond to $-1.2-1.2$ in phase shift (radian). (a)

(b)

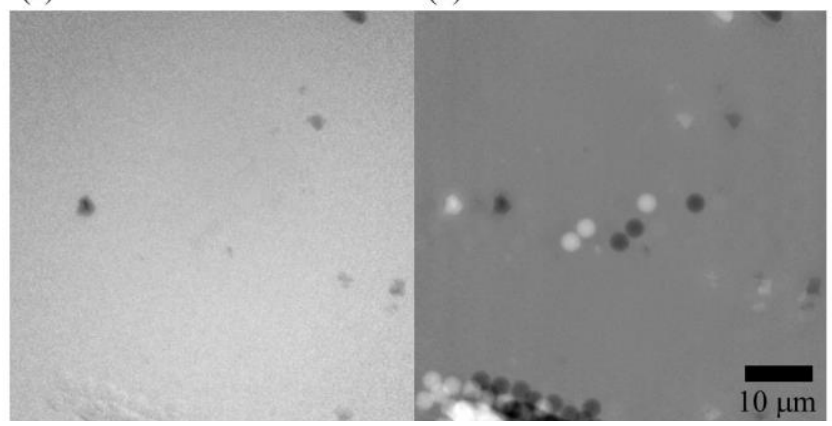

Figure. 2. Comparison of observed image of polystyrene spheres (diameter: $3 \mu \mathrm{m}$ ). (a) Absorption image and (b) twin phase image. The gray scales of the images correspond to (a) $0.95-1.01$ in transmittance and (b) $-1-1$ in

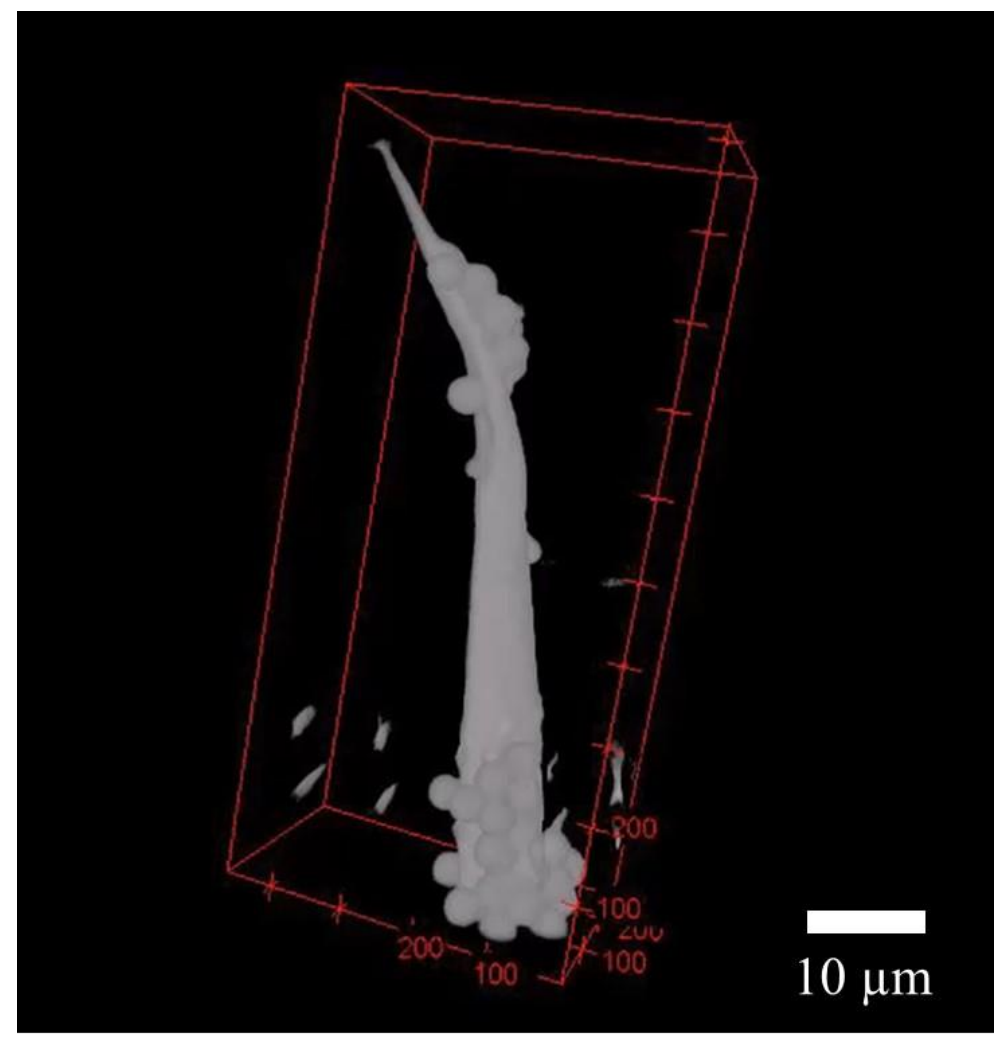

Figure. 3. X-ray phase-CT image (volume rendering) of polystyrene spheres (diameter: $3 \mu \mathrm{m}$ ) on a plastic fiber obtained by $360 \mathrm{CT}$ projections. Single projection image was obtained by phase stepping measurement of 6 step with each exposure time of $20 \mathrm{~s}$. 